\title{
Hiperplasia virginal mamaria asociado a hiperplasia pseudoangiomatosa del estroma mamario difuso
}

\author{
Virginal breast hypertrophy associated to difuse \\ pseudoangiomatous stromal hyperplasia of the breast
}

\section{Oscar Alejandro Bonilla Sepúlveda' ${ }^{1 \otimes c} \underline{\text { chac }}$}

1. Mastólogo.Clínica del Prado. Medellin, Colombia.

Este trabajo cumple con las siguientes características editoriales

Fecha correspondencia: Recibido: septiembre 24 de 2014. Revisado: septiembre 9 de 2015. Aceptado: septimbre 17 de 2015.

Forma de citar

Bonilla-Sepúlveda OA.

Hiperplasia virginal mamaria asociado a hiperplasia pseudoangiomatosa del estroma mamario difuso. Rev CES Med 2016. 30(1): 122-128.

Open Acces

(C) Derecho de autor

Licencia creative commons

Ética de publicaciones

Revisión por pares

Gestión por Open Journal System

ISSN 0129-8705

ISSN-e 2215-9177

$\underline{\text { Comparte }}$

CBS MEDICINA

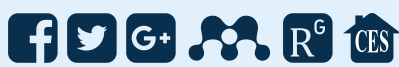

\section{Resumen}

Se presenta el caso de una mujer de 20 años de edad con crecimiento mamario severo bilateral tipo gigantomastia, de tres meses de evolución, quien fue valorada inicialmente en hospital de primer nivel de complejidad, donde se le hizo diagnóstico de mastitis aguda, la cual no tuvo respuesta a los antibióticos y cuya biopsia de mama fue sospechosa para angiosarcoma mamario, por lo cual remiten a un tercer nivel. Allí fue valorada por el grupo de mastología que reorienta el diagnóstico luego de exámenes complementarios, documentándose así una poco frecuente asociación entre hipertrofia virginal mamaria e hiperplasia pseudoangiomatosa del estroma mamario difusa. Se revisa la presentación clínica de ambas entidades y su diagnóstico diferencial.

Palabras clave: Hiperplasia, Hipertrofia, Mesodermo, Mama.

\begin{abstract}
Presents a woman of 20 years old with severe bilateral mammary growth rate type gigantomastia with three months of duration. Initially valued and diagnosed at first level as an acute mastitis, which didn't respond to antibiotics. A mammary biopsy was suspicious for breast angiosarcoma, and refered, valued by mastology that reorients the diagnosis after further examination and biopsy, and documenting a rare association between virginal breast hypertrophy and difuse pseudoangiomatous hyperplasia of the mammary stroma, which together with a good diagnosis could receive appropriate treatment. The clinical presentation of the diseases and their differential diagnosis is reviewed.
\end{abstract}

Keywords: Hyperplasia, Hypertrophy, Mesoderm, Breast.

\section{Introducción}

La hipertrofia virginal mamaria tiene algunos términos descriptivos (por ejemplo, hipertrofia juvenil, hipertrofia virginal, macromastia juvenil, gigantomastia, entre otros) y fue descrita por primera vez por Durston en 1669 (1). 
Se trata de un trastorno raro y benigno, de etiología incierta, en la que se produce un rápido y contínuo crecimiento del tejido mamario durante la pubertad; a menudo hay un período de seis meses de crecimiento extremo y luego un período más lento pero sostenido. Ocurre más comúnmente en las niñas entre ocho y 16 años de edad.
Se trata de un trastorno raro y benigno, de etiología incierta (2), en la que se produce un rápido y contínuo crecimiento del tejido mamario durante la pubertad; a menudo hay un período de seis meses de crecimiento extremo y luego un período más lento pero sostenido. Ocurre más comúnmente en las niñas entre ocho y 16 años de edad; se desarrolla de forma esporádica, aunque se han reportado casos familiares ().

El crecimiento excesivo suele ser bilateral, sin embargo se han reportado casos unilaterales (4-7). Puede causar varios problemas clínicos como dolor en pecho, espalda y cuello, así como dilatación de las venas superficiales y ulceración de la piel. También puede causar alteraciones psicológicas y cosméticas (ㅇ).

El mecanismo subyacente que causa la hipertrofia de la mama no ha sido dilucidado. Una teoría propuesta es una hipersensibilidad de los órganos diana a niveles normales de hormonas gonadales (9). Una hipótesis alternativa es que hay aumento de la actividad hormonal; sin embargo, estudios previos muestran niveles normales de estrógeno, progesterona, gonadotropinas y la hormona del crecimiento (2).

Bajo esta hipótesis hormonal se han realizado intentos de controlar esta condición, utilizando medicamentos como tamoxifeno, danazol o bromocriptina (10), aunque la seguridad y eficacia de la farmacoterapia es desconocida actualmente. Por esta razón, a la mayoría de las adolescentes diagnosticadas con esta condición se les recomienda someterse a mamoplastia de reducción, una vez que se estabiliza el tamaño de la mama, siendo ésta la opción que tiene menores tasas de recurrencia (11).

La hiperplasia estromal pseudoangiomatosa de la mama (por sus siglas en inglés PASH), fue descrita por primera vez en 1986 por Vuitch et al. (12). Es una proliferación benigna poco frecuente de células estromales mesenquimales, con formaciones de ranura irregulares parecidas a estructuras angiomatosas $(13,14)$. En la mayoría de los casos es un hallazgo microscópico focal en biopsias de mama realizadas por enfermedades benignas o malignas.

Se puede presentar en forma difusa o nodular como una masa palpable intramamaria indolora, casi exclusivamente en mujeres premenopáusicas (2). Tiende a crecer con el tiempo y la mayoría de los casos se pueden curar mediante la extirpación y el pronóstico es bueno (15).

La etiología exacta y la patogénesis de esta lesión tumoral siguen siendo desconocidas, aunque ha sido postulada una respuesta proliferativa de mio-fibroblastos a estímulos hormonales (16). Histológicamente, se encuentran hendiduras ramificadas, bordeadas de miofibroblastos que se pueden anastomosar y confundirse con espacios vasculares, lo que lleva a un diagnóstico erróneo de angiosarcoma (17).

\section{Caso clínico}

Se trataba de una paciente de sexo femenino, 20 años, quien fue atendida en hospital local de un municipio del departamento de Antioquia (Colombia), por un cuadro clínico de tres meses de evolución de aumento severo del volumen mamario en forma bilateral, sin masa palpable, asociado a leve eritema. La paciente no refería mastodinia, prurito, telorrea o adenopatías.

Se le realizaron exámenes paraclínicos que reportaron lo siguiente: hemoglobina 10,3 mg \%; glóbulos blancos $8160 \mathrm{~mm}^{3}$; neutrófilos $48 \%$, linfocitos $24 \%$, plaquetas 140000 (disminuido), tiempo protrombina 22 segundos (normal), tiempo parcial 


\section{Ambas mamas con} hipertrofia severa pero con predominio de la mama derecha, con volumen aproximado de 1500 cc y catalogada como gigantomastia (clasificación de Lalardrie-Jouglard), piel tensa, finas estrías, prominente red venosa superficial, congestión vascular y leve eritema. tromboplastina tisular: 41 segundos (normal), prolactina: 200 (elevado), prueba de embarazo negativa, radiografía de tórax normal.

La ecografía mamaria reportó marcado edema de parénquima glandular, con aumento del volumen asociado a congestión linfática bilateral sin evidencia de imágenes focales de aspecto sólido o quístico, por lo cual se le realizó diagnóstico de mastitis, requiriendo hospitalización por ocho días para manejo antibiótico con clindamicina, oxacilina, pero sin mejoría clínica.

Se decide entonces tomar biopsia cortante eco guiada bilateral de mamas y se envían las muestras a centro patológico local con resultado de proliferación vascular en clasificación (¿angiosarcoma?), para lo cual se solicita inmunohistoquímica y se remite a tercer nivel en Medellín para evaluación y manejo por equipo de mastología.

Al ingreso se encontró una paciente tranquila pero ansiosa, muy preocupada por la alteración de su imagen corporal, hemodinámicamente estable, tensión arterial de 110/70, pulso de 80 latidos por minuto, afebril, ambas mamas con hipertrofia severa pero con predominio de la mama derecha, con volumen aproximado de $1500 \mathrm{cc} \mathrm{y} \mathrm{ca-}$ talogada como gigantomastia (clasificación de Lalardrie-Jouglard), piel tensa, finas estrías, prominente red venosa superficial, congestión vascular y leve eritema, sin calor, sin signos de infección local, adenopatías axilares, telorrea u otras alteraciones (figura 1).
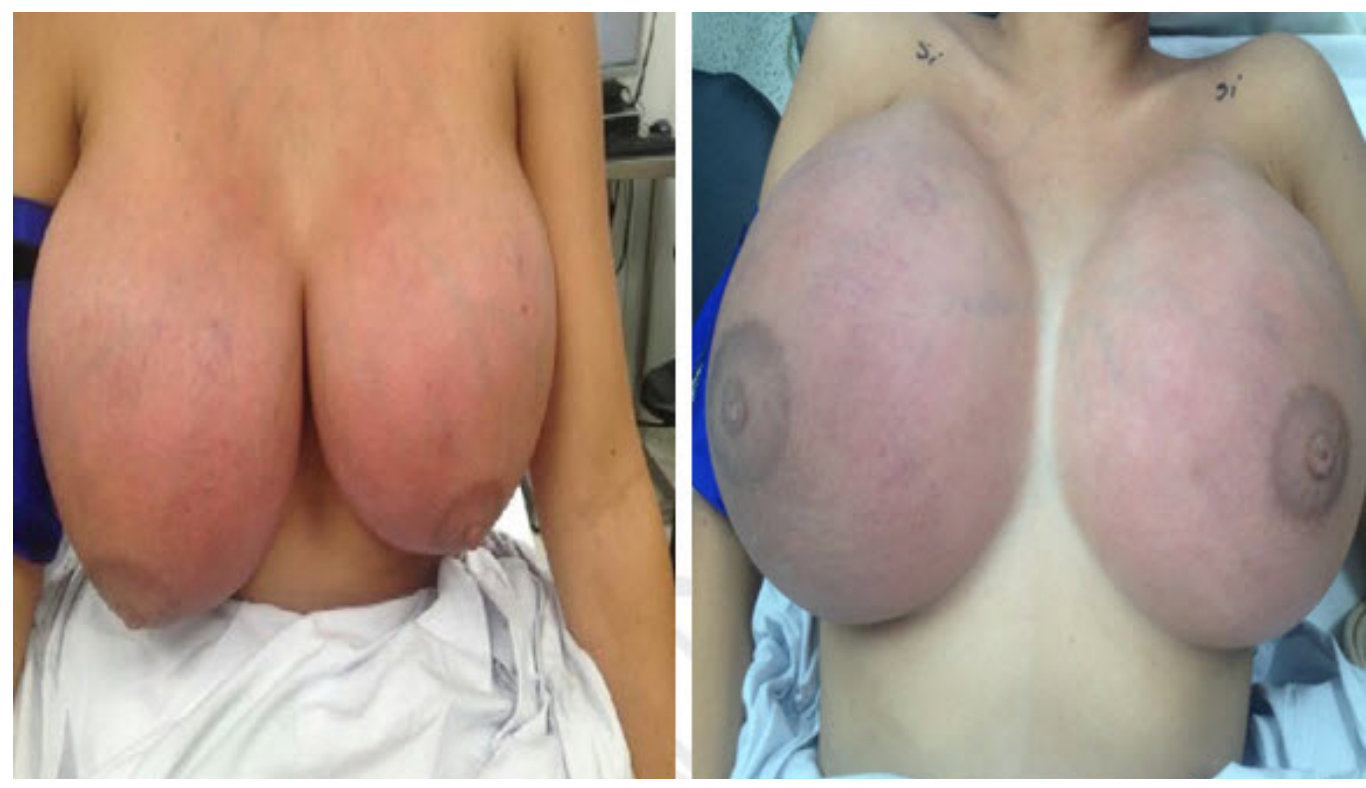

Figura 1. Mamas hipertróficas severas bilaterales tipo gigantomastia con predominio de la mama derecha, piel tensa y red venosa superficial prominente

Como antecedentes gineco-obstétricos se reportaba, hijos: 0, aborto: 0, amenorrea de 90 días, vida sexual activa, planificando con preservativo, aunque anteriormente con método inyectable mensual, el cual fue suspendido hace cinco meses, ultima citología hace un año la cual fue reportada como normal, sin antecedentes patológicos o familiares relevantes.

Con diagnóstico de gigantomastia en estudio con biopsias no conclusivas, hiperprolactinemia con amenorrea secundaria, anemia y trombocitopenia leve, se hospitaliza 
Enero - Junio 2016 - Pág 125

El volumen ideal de mama es de 250 a 300 $\mathrm{mm}^{3}$, por lo que un volumen entre 400 y 600 $\mathrm{mm}^{3}$ es considerado como hipertrofia moderada y más de 1500 $\mathrm{mm}^{3}$ como gigantomastia. para realizar exámenes complementarios. La mamografía reportó cambios hiperplásicos bilaterales de predominio derecho, en cuadrantes superiores externos, sugestivo de hiperplasia sin alteración tumoral. La resonancia magnética contrastada de silla turca reportó neuro-adeno hipófisis y quiasma normales, no se identificaron lesiones hipocaptantes sugestivas de adenoma hipofisiario.

Se hace entonces nueva biopsia de tipo incisional y a cielo abierto bilateral, además de relectura de las placas de la biopsia tomada en unidad local, encontrando histológicamente un patrón característico de espacios en forma de ranuras (pseudoangiomatoso) formando anastomosis alineadas, con proliferación de miofibroblastos en el estroma y que no contienen hematíes.

Con estos hallazgos se realiza un diagnóstico patológico final de hiperplasia pseudoangiomatosa del estroma mamario difuso, clínicamente asociado a una hipertrofia virginal (juvenil) mamaria, que requieren valoración concomitante por endocrinología, psicología y cirugía plástica, que como medida final decide programarla para mamoplastia de reducción bilateral con patrón de Wise.

\section{Discusión}

La hipertrofia juvenil de la mama es un trastorno poco común. Bauer et al. reportan que constituye el $12,5 \%$, de todas las enfermedades mamarias en adolescentes, mientras la gigantomastia tiene una prevalencia de 1 por cada 25000 mujeres (1). Clínicamente, se presenta como un rápido crecimiento de la mama en un período de tres a seis, seguido por un crecimiento lento y constante (4).

Se considera que el volumen ideal de mama es de 250 a $300 \mathrm{~mm}^{3}$, por lo que un volumen entre 400 y $600 \mathrm{~mm}^{3}$ es considerado como hipertrofia moderada y más de $1500 \mathrm{~mm}^{3}$ como gigantomastia (18). De esta última condición se han reportado casos de hasta 13,5 a $22,5 \mathrm{~kg}$ (19). En el caso descrito, la tasa de crecimiento fue rápida durante los primeros tres meses y el tamaño final llegó a rangos de gigantomastia.

Clínicamente se encuentran senos generalmente pendulares, difusamente firmes, sin ningún tipo de masas, se pueden observar dilatación de las venas superficiales o ulceraciones de la piel. La enfermedad puede causar mastodinia, dolor de espalda o del cuello, usualmente asociados a trastornos de imagen corporal y autoestima.

La hipertrofia juvenil de la mama es un diagnóstico de exclusión y siempre se deben descartar otras causas como la pseudo-gigantomastia asociada con la obesidad, tumores fibroepiteliales de la mama como tumor phyllodes y fibroadenomas, hipertrofia grávidica de la mama, tumores malignos, tales como linfomas y los sarcomas de mama (20).

También se debe considerar la hipertrofia secundaria a trastornos endocrinos, tales como pubertad precoz verdadera resultante de lesiones hipotalámicas, precocidad constitucional, displasia fibrosa poliostótica, pubertad resultante de tumor de células de la granulosa ovárica, quistes foliculares, tumores suprarrenales y la exposición a una variedad de hormonas, incluyendo gonadotropinas, estrógenos, testosterona y corticosteroides (2).

Por su parte, la hiperplasia estromal pseudoangiomatosa es una lesión proliferativa benigna del estroma mamario, con una prevalencia de 0,4 \% (ㄷ). Morita et al., describen que la enfermedad podía estar asociada a otras enfermedades como mastopatía $(14,1 \%)$, fibroadenoma $(0,9 \%)$, y carcinoma (6,7 \%) (21). 
Los hallazgos histológicos más asociados de la hiperplasia estromal pseudoangiomatosa de la mama son los cambios fibroquísticos, hiperplasia ductal sin atipia, adenosis, metaplasia apocrina, cambios fibroadenomatosos, cambios similares a ginecomastia, pero pocas veces relacionado a hiperplasia mamaria.
Puede no tener representación clínica como en la variedad difusa y hallarse de forma accidental en biopsias aisladas de mama, o manifestarse como una lesión nodular palpable, única o múltiple, sin dolor, móvil, semiblanda, que puede ser bilateral y tan grande como $7 \mathrm{~cm}$ de diámetro (19). En el caso descrito la presentación fue la de un hallazgo patológico sin masa palpable.

El diagnóstico diferencial más importante es con el angiosarcoma de bajo grado, en el que se observan hematíes en los espacios vasculares y están completamente rodeada por células endoteliales atípicas y con actividad mitótica (22).

Los hallazgos histológicos más asociados de la hiperplasia estromal pseudoangiomatosa de la mama son los cambios fibroquísticos, hiperplasia ductal sin atipia, adenosis, metaplasia apocrina, cambios fibroadenomatosos, cambios similares a ginecomastia, pero pocas veces relacionado a hiperplasia mamaria severa (gigantomastia) en un mismo contexto clínico. No hay reportes de características similares de este caso documentadas en la literatura (23).

\section{Conclusión}

La hiperplasia estromal pseudoangiomatosa y la hipertrofia mamaria virginal son dos enfermedades mamarias poco frecuentes, que pueden presentarse concomitantemente. Ambas son de carácter benigno y etiología desconocida, aunque se ha planteado hipótesis hormonales. El estudio histológico puede confundirse con un tumor vascular, especialmente angiosarcoma de bajo grado; además, por la edad de las pacientes y su repercusión emocional se requiere de manejo por un grupo multidisciplinario en mastología, patología, endocrinología, cirugía plástica y psicología.

\section{Consideraciones éticas}

En la elaboración del presente documento no se publican nombres, documentos de identidad o cualquier otra información que pudiera vincular a las pacientes y que pudieran ser objeto de violación de la privacidad. Este trabajo cumple toda la normatividad internacional para la investigación médica (Declaración de Helsinki y el Informe Belmont), según la Resolución 8430 de 1993 del Ministerio de Salud de la Republica de Colombia (24).

\section{Agradecimientos}

A los doctores Carlos Alberto Restrepo Ramírez especialista en mastología, Gustavo Matute Turizo especialista en patología y a los especialista en radiología Gloria Mesa y Aurelio González Calle que hicieron parte del grupo de médicos tratantes.

Conflicto de intereses: Ninguno

\section{Bibliografía}

1. Bauer BS, Jones KM, Talbot CW. Mammary masses in the adolescent female. Surg Gynecol Obstet 1987; 165: 63-65. link

2. Kupfer D, Dingman D, Broadbent R. Juvenile breast hypertrophy: report of a familial pattern and review of the literature. Plast Reconstr Surg 1992; 90: $303-$ 309. link

3. Baker SB, Burkey BA, Thornton P, LaRossa D. Juvenile gigantomastia: presentation of four cases and review of the literature. Ann Plast Surg 2001; 5: 517-526. link 
4. Neinstein LS. Breast disease in adolescents and young women. Pediatr Clin North Am 1999; 46: 607-629. link

5. Templeman C, Hertweck SP. Breast disorders in the pediatric and adolescent patient. Obstet Gynecol Clin North Am 2000; 27: 19-34. link

6. Netscher DT, Mosharrafa AM, Laucirica R. Massive asymmetric virginal breast hypertrophy. South Med J 1996; 89: 434. link

7. Agaoglu G, Ozgur F, Erk Y. Unilateral virginal breast hypertrophy. Ann Plast Surg 2000; 45: 451-453. link

8. Dehner LP, Hill DA, Deschryver K. Pathology of the breast in children, adolescents, and young adults.Semin Diagn Pathol 1999; 16: 235-247. link

9. Uribe Barreto A. Juvenile mammary hypertrophy. Plast Reconstr Surg 1991; 87(3):583-584. link

10. Sperling RL, Gold JJ. Use of an anti-estrogen after a reduction mammaplasty to prevent recurrence of virginal hypertrophy of breasts. Case report. Plast Reconstr Surg 1973; 52(4):439-442. link

11. Arscott GD, Craig HR, Gabay L. Failure of bromocriptine therapy to control juvenile mammary hypertrophy. Br J Plast Surg 2001;54 (8):720-723. link

12. Vuitch MF, Rosen PP, Erlandson RA. Pseudoangiomatous hyperplasia of mammary stroma. Hum Pathol 1986; 17:185-91. link

13. Gow KW, Mayfi eld JK, Lloyd D, Shehata BM. Pseudoangiomatous stromal hyperplasia of the breast in two adolescent females. Am Surg. 2004; 70(7):605-8. link

14. Castro CY, Whitman GJ, Sahin AA Pseudoangiomatous stromal hyperplasia of the breast. Am J Clin Oncol. 2002; 25(2):213-6. link

15. McCluggage WG, Allen M, Anderson NH. Fine needle aspiration cytology of mammary pseudoangiomatous stromal hyperplasia. A case report. Acta Cytol 1999; 43:1147-9. link

16. Vicandi B, Jimenez-Heff ernan JA, Lopez-Ferrer P, Ortega L, Viguer JM. Nodular pseudoangiomatous stromal hyperplasia of the breast. Cytologic features. Acta Cytol. 1998; 42(2):335-41. link

17. Ibrahim RE, Sciotto CG, Weidner N. Pseudoangiomatous hyperplasia of mammary stroma. Some observations regarding its clinicopathologic spectrum. Cancer 1989; 63:1154-60. link

18. Polger MR, Denison CM, Lester S, Meyer GE. Pseudoangiomatous stromal hyperplasia: mammographic and sonographic appearances. Am J Roentgenol 1996; 166:349-52. link

19. Moriya T, Endoh M, Semba S: Pseudoangiomatous stromal hyperplasia (PASH). Byori to Rinsyo 19:518-520, 2001. link 
20. Di Noto A, Pacheco BP, Vicala RE. Two cases of breastlymphoma mimicking juvenile hypertrophy. J Pediatr Adolesc Gynecol 1999; 12: 33-35. link

21. Oberman HA. Breast lesions in the adolescent female. Ann Pathol 1979; 1: 175. link

22. Cohen MA, Morris EA, Rosen PP, Dershaw DD, Liberman L,Abramson AF. Pseudoangiomatous stromal hyperplasia: mammographic, sonographic and clinical patterns. Radiology 1996; 198:117-120. link

23. Ferreira M, Albarracin CT, Resetkova E. Pseudoangiomatous stromal hyperplasia tumor: a clinical, radiologic and pathologic study of 26 cases. Mod Pathol. 2008 Feb;21(2):201-7. Epub 2007 Dec 14. link

24. Ministerio de Salud. Resolución No. 008430 de 1993. Normas Científicas, Técnicas y Administrativas para la investigación en salud. Santa Fe de Bogotá. 1993. p.1. link 